\title{
Large Deviations and Lifshitz Singularity of the Integrated Density of States of Random Hamiltonians
}

\author{
Werner Kirsch and Fabio Martinelli* \\ Institut für Mathematik, Ruhr-Universität Bochum, Postfach 102148, D-4630 Bochum 1, Federal \\ Republic of Germany
}

\begin{abstract}
We consider the integrated density of states (IDS) $\rho_{\infty}(\lambda)$ of random Hamiltonian $H_{\omega}=-\Delta+V_{\omega}, V_{\omega}$ being a random field on $\mathbb{R}^{d}$ which satisfies a mixing condition. We prove that the probability of large fluctuations of the finite volume IDS $|\Lambda|^{-1} \rho\left(\lambda, H_{\Lambda}(\omega)\right), \Lambda \subset \mathbb{R}^{d}$, around the thermodynamic limit $\rho_{\infty}(\lambda)$ is bounded from above by $\exp \{-k|\Lambda|\}, k>0$. In this case $\rho_{\infty}(\lambda)$ can be recovered from a variational principle. Furthermore we show the existence of a Lifshitztype of singularity of $\rho_{\infty}(\lambda)$ as $\lambda \rightarrow 0^{+}$in the case where $V_{\omega}$ is non-negative. More precisely we prove the following bound: $\rho_{\infty}(\lambda) \leqq \exp \left(-k \lambda^{-d / 2}\right)$ as $\lambda \rightarrow 0^{+} k>0$. This last result is then discussed in some examples.
\end{abstract}

\section{Section 1. Introduction}

Let $V_{\omega}(x), x \in \mathbb{R}^{d}$, be a metrically transitive random field on $\mathbb{R}^{d}$ and let $H_{\omega}$ be the (formal) random Hamiltonian $H_{\omega}=-\Delta+V_{\omega}$. Under very weak assumptions on $V_{\omega}($ see $[11]), H_{\omega}$ is essentially selfadjoint on $C_{0}^{\infty}\left(\mathbb{R}^{d}\right) \subset L^{2}\left(\mathbb{R}^{d}\right)$ and it is used to model physical systems in presence of disorder, e.g. a particle in a crystal with random impurities. The integrated density of states (IDS) $\rho_{\infty}(\lambda), \lambda \in \mathbb{R}$, plays an important role in the physics of such systems. The IDS $\rho_{\infty}(\lambda)$ is defined as follows:

$$
\rho_{\infty}(\lambda)=\lim _{\Lambda_{n} \uparrow \mathbb{R}^{d}} \frac{1}{\left|\Lambda_{n}\right|} \rho\left(\lambda, H_{\Lambda_{n}}(\omega)\right) .
$$

Here $\left\{\Lambda_{n}\right\}_{n \in \mathbb{N}}$ is a sequence of hypercubes increasing to $\mathbb{R}^{d},|\cdot|$ denotes the Lebesgue measure, and $\rho\left(\lambda, H_{A}(\omega)\right)$ is the number of eigenvalues less than $\lambda$ of $H(\omega)$ restricted to $L^{2}(\Lambda)$ with suitable boundary conditions. It can be proved in great generality that with probability one $\rho_{\infty}(\lambda)$ exists for all $\lambda \in Q$ and that it is independent of $\omega$ and of the chosen boundary conditions. Furthermore the measure on $\mathbb{R}$ whose distribution function is $\rho_{\infty}$ has support on the almost surely constant spectrum of $H_{\omega}$. (See e.g. [1], [10] and references therein.) In the next section we study the large

* On leave of absence from Istituto di Fisica, Università di Roma, ITALY, G.N.F.M. C.N.R. 
fluctuations of the finite volume IDS $|\Lambda|^{-1} \rho\left(\lambda, H_{\Lambda}(\omega)\right)$ around the thermodynamic limit $\rho_{\infty}(\lambda)$. Following a recent method proposed by Ellis [5] we show that under a mixing condition $(\varphi$-mixing) the probability of these fluctuations is bounded by $\exp (-|\Lambda| k)$ for some positive constant $k$. This is proved in Theorem 2 .

In particular we show that $|\Lambda|^{-1} \rho\left(\lambda, H_{\Lambda}(\omega)\right)$ converge geometrically to $\rho_{\infty}(\lambda)$ as $\Lambda \uparrow \mathbb{R}^{d}$ in the sense that:

$$
P\left(\left.|| \Lambda_{n}\right|^{-1} \rho\left(\lambda, H_{\Lambda_{n}}(\omega)\right)-\rho_{\infty}(\lambda) \mid>\delta\right) \leqq e^{-\left|\Lambda_{n}\right| M(\delta)}
$$

for all $\delta>0$ and $n$ sufficiently large, where $M(\delta)$ is a positive constant. If in addition the random field $V_{\omega}$ satisfies a stronger independence assumption than $\varphi$-mixing, the above estimate is shown to be optimal in the limit $\Lambda \uparrow \mathbb{R}^{d}$ at least for elementary events of the form:

$$
\left\{\omega \in \Omega ;|\Lambda|^{-1} \rho\left(\lambda, H_{\Lambda}(\omega)\right) \geqq x\right\} .
$$

A typical example in which this independence condition is fulfilled is the Anderson model: $H(\omega)=-\Delta_{d}+V_{\omega}$ on $l^{2}\left(Z^{d}\right),-\Delta_{d}$ being the discrete Laplacian and $\left\{V_{\omega}(i)\right\}_{i \in Z^{d}}$ iid random variables.

A different problem is the behaviour of $\rho_{\infty}(\lambda)$ as $\lambda \rightarrow 0^{+}$in the case where $V_{\omega}$ is a non-negative random field satisfying a $\varphi$-mixing condition.

In the last section, using an exponential estimate for the probability of large deviations for weakly dependent random variables proved in Sect. 2 and a rigorous version of an argument due to Lifshitz [14], we prove an upper bound on $\rho_{\infty}(\lambda)$ of the form:

$$
\rho_{\infty}(\lambda) \leqq e^{-k \lambda-d / 2}
$$

as $\lambda \rightarrow 0^{+}$for some $k>0$.

Under an additional independence assumption we also give a lower bound of the same type but with a different constant $k^{\prime}$. This result is then discussed in the case when i) $V_{\omega}$ is a positive function of a Gaussian random field. ii) $V_{\omega}=\sum_{i \in Z^{d}} \varphi_{i}(\omega, x-i)$ with $\left\{\varphi_{i}(\omega)\right\}_{i \in Z^{d}}$ iid random variables with values in $l^{1}\left(L^{p}\right)$, the Banach space of all measurable functions $f: \mathbb{R}^{d} \rightarrow \mathbb{R}$ such that: $\left.\left.\sum_{i \in Z^{d}}\left|\int_{C_{0}}\right| f(x-i)\right|^{p} d x\right|^{p}<+\infty, C_{0}$ being the unit cell in $\mathbb{R}^{d}$ around $x=0$. The singular behaviour (2), known as the Lifshitz singularity [14], was already proved by means of Wiener integrals by several authors (see e.g. [6], [16], [17], [18]) for the case in which $V_{\omega}(x)=\sum_{i} \varphi\left(x-x_{i}(\omega)\right)$, where $\varphi$ is a positive function on $\mathbb{R}^{d}$ with sufficient decay at infinity and $\left\{x_{i}(\omega)\right\}_{i \in \mathbb{N}}$ is a realization of the Poisson random field on $\mathbb{R}^{d}$. In this case it is even possible to compute exactly

$$
\lim _{\lambda \rightarrow 0^{+}}-\lambda^{d / 2} \ln \rho_{\infty}(\lambda)=k
$$

using the Wiener sausage techniques developed by Donsker and Varadhan [4]. We also refer to [21] for a discussion of the same problem for the Anderson model. The reader mainly interested in the Lifshitz exponent may skip Sect. 2 with the exception of Lemma 2, the proof of which is needed for the proof of Theorem 4. 
Notations and Assumptions. Throughout all this paper $\Lambda$ will denote an arbitrary bounded hypercube in $\mathbb{R}^{d}$ and $|\Lambda|$ its Lebesgue measure. On the space $L^{2}(\Lambda)$ we will consider the selfadjoint operators with compact resolvent $H_{A}^{N}, H_{A}^{D}$ defined as form sum by:

$$
\begin{aligned}
& H_{A}^{N}=-\Delta_{A}^{N}+V, \\
& H_{A}^{D}=-\Delta_{A}^{D}+V,
\end{aligned}
$$

where $-\Delta_{A}^{N},-\Delta_{A}^{D}$ are the Neumann and Dirichlet Laplacian respectively (see e.g. Reed-Simon IV [19]) and $V \in L^{p}(\Lambda), p=1$ if $d=1, p>1$ if $d=2, p=d / 2$ if $d \geqq 3$. We will denote by $\left\{\lambda_{k}\left(H_{A}^{N}\right)\right\}_{k \in \mathbb{N}} \cdot\left\{\lambda_{k}\left(H_{A}^{D}\right)\right\}_{k \in \mathbb{N}}$ their eigenvalues (counting multiplicity) and by $\rho\left(\lambda, H_{\Lambda}^{N}\right), \rho\left(\lambda, H_{\Lambda}^{D}\right)$ the positive nondecreasing functions on $\mathbb{R}$ defined by:

$$
\rho\left(\lambda, H_{\Lambda}^{N}\right)=\left\{k \in \mathbb{N} ; \lambda_{k}\left(K_{\Lambda}^{N}\right)<\lambda\right\},
$$

and analogously for $\rho\left(\lambda, H_{A}^{D}\right)$. Finally we will denote by $C_{0}$ the unit cell in $\mathbb{R}^{d}$ around $x=0$, and by $C_{i}$ the set $C_{0}+i, i \in Z^{d}$.

Now $V_{\omega}(x), x \in \mathbb{R}^{d}$, be a measurable random field on $\mathbb{R}^{d}$ on which we assume:

(A) i) There exists on the probability space $(\Omega, \mathscr{F}, P)$ a group of measurepreserving metrically transitive transformations $\left\{T_{i}\right\}_{i \in I} I=\mathbb{R}^{d}$ or $I=Z^{d}$, such that $V_{\omega}(x+i)=V_{T_{i} \omega}(x) \forall x \in \mathbb{R}^{d}, \forall i \in I$.

ii) $E\left\{\int_{C_{0}}\left|V_{\omega}(x)\right|^{p} d x\right\}<+\infty$, where $p>\max (2, d / 2)$ and $E\{\cdot\}$ denotes the expectation with respect to the measure $P$.

iii) Let $V_{\omega}^{-}(x)=\min \left(0, V_{\omega}(x)\right)$; then $\int_{C_{i}}\left|V_{\omega}^{-}(x)\right|^{q} d x \leqq C<+\infty$ for some $q>\max (2, d / 2)$ and a positive constant $C$ independent of $i \in Z^{d}$ and $\omega \in \Omega$.

(B) For any $\Lambda \subset \mathbb{R}^{d}$ let $\Sigma_{\Lambda}$ be the $\sigma$-algebra generated by $V_{\omega}(x), x \in \Lambda$, and let $f, g$ be two arbitrary random variables on $\Omega$ such that:

i) $|g|_{\infty}<+\infty, E\{|f|\}<+\infty$,

ii) $g$ is $\Sigma_{\Lambda_{1}}$-measurable, $f$ is $\Sigma_{\Lambda_{2}}$-measurable, where $\Lambda_{1}, \Lambda_{2}$ are bounded subsets of $\mathbb{R}^{d}$ with $\Lambda_{1} \cap \Lambda_{2}=\phi$. Then:

$$
|E\{f \cdot g\}-E(f) E(g)| \leqq|g|_{\infty} E\{|f|\} \varphi\left(d\left(\Lambda_{1}, \Lambda_{2}\right)\right)
$$

with $\varphi(x) \rightarrow 0$ as $x \rightarrow+\infty$. Here $d\left(\Lambda_{1}, \Lambda_{2}\right)$ denotes the Euclidean distance between $\Lambda_{1}$ and $\Lambda_{2}$.

It is known (see e.g. Billingsley [3]) that (B) holds if the random field $V_{\omega}$ satisfies a $\varphi$-mixing condition. Let now $H_{A}^{D}(\omega)=-\Delta_{A}^{D}+V_{\omega}$ and $H_{\Lambda}^{N}(\omega)=-\Delta_{A}^{N}+V_{\omega}$. Using (A) it is possible to prove (see e.g. [10]) that the following limits exist for almost all $\omega$ and all $\lambda \in Q$ :

$$
\lim _{\Lambda_{n} \uparrow \mathbb{R}^{d}} \frac{1}{\left|\Lambda_{n}\right|} \rho\left(\lambda, H_{\Lambda_{n}}^{D}(\omega)\right)=\rho_{\infty}(\lambda)=\lim _{\Lambda_{n} \uparrow \mathbb{R}^{d}} \frac{1}{\left|\Lambda_{n}\right|} \rho\left(\lambda, H_{\Lambda_{n}}^{N}(\omega)\right),
$$

where $\left\{\Lambda_{n}\right\}_{n \in \mathbb{N}}$ is a sequence of cubes increasing to $\mathbb{R}^{d}$ and $\rho_{\infty}(\lambda)$ is a nonrandom, nondecreasing function on $\mathbb{R}$. Furthermore for any bounded cube $\Lambda \subset \mathbb{B}^{d}$ and any 
$\lambda \in \mathbb{R} \rho_{\infty}(\lambda)$ satisfies:

$$
|\Lambda|^{-1} E\left\{\rho\left(\lambda, H_{\Lambda}^{D}(\omega)\right)\right\} \leqq \rho_{\infty}(\lambda) \leqq|\Lambda|^{-1} E\left\{\rho\left(\lambda, H_{\Lambda}^{N}(\omega)\right)\right\} .
$$

The function $\rho_{\infty}(\lambda)$ is called the integrated density of states (IDS) for the selfadjoint operator $H_{\omega}=-\Delta+V_{\omega}$. Finally we define the positive measures on $\mathbb{R} \mu_{\Lambda}^{D}(\omega), \mu_{\Lambda}^{N}(\omega)$, $\mu_{\infty}$ whose distribution functions are $|\Lambda|^{-1} \rho\left(\lambda, H_{A}^{D}(\omega)\right),|\Lambda|^{-1} \rho\left(\lambda, H_{A}^{N}(\omega)\right)$ and $\rho_{\infty}(\lambda)$ respectively.

Remark 1. It follows from (A) that:

i) the measures $\mu_{\Lambda}^{D}(\omega), \mu_{\Lambda}^{N}(\omega)$ are locally bounded uniformly in $\omega$ and in $\Lambda \supset C_{0}$.

ii) There exists an $a_{0},+\infty>a_{0}>-\infty$, such that $\mu_{\Lambda}^{D}(\omega)=\mu_{\Lambda}^{N}(\omega)=0$ on $(-\infty$, $a_{0}$ ) for a.e. $\omega \in \Omega$ and $\Lambda \supset C_{0}$.

\section{Section 2. A Large Deviation Result}

In this section we examine how the limit (3) is attained in that we provide an upper bound on the probability of large fluctuations of the measure $\mu_{\Lambda}^{D}(\omega)$ around the thermodynamic limit $\mu_{\infty}$. In order to simplify the discussion we restrict both $\mu_{\Lambda}^{D}(\omega)$ and $\mu_{\infty}$ to a bounded interval $\left[a_{0}, b\right]$ where $a_{0}$ is defined in Remark 1 and $b>a_{0}$ is a continuity point of $\rho_{\infty}(\lambda)$. With this choice $\int^{b} f(\lambda) d \mu_{\Lambda}^{D}(\omega, \lambda) \rightarrow \int_{a_{0}}^{b} f(\lambda) d \mu_{\infty}(\lambda)$, as $\Lambda \uparrow \mathbb{R}^{d}$ for any continuous function $f$ on $\left[a_{0}, b\right]$. For notational convenience we denote $\left.\mu_{\Lambda}^{D}(\omega)\right|_{\left[a_{0}, b\right]}$ again by $\mu_{\Lambda}^{D}(\omega)$ and analogously for $\mu_{\infty}$. By Remark 1 for any $\omega \in \Omega$ and any $\Lambda \supset C_{0}, \mu_{\Lambda}^{D}(\omega)$ and $\mu_{\infty}$ are elements of $M_{a_{0}, b}^{+, k}$, the space of positive Borel measures on $\left[a_{0}, b\right]$ with total mass less than a sufficiently large constant $k$. We equip $M_{a_{0}, b}^{+, k}$ with the weak-*-topology and define for a measurable set $A \subset M_{a_{0}, b}^{+, k}$ :

$$
\widetilde{P}_{\Lambda}(A)=P\left(\left\{\omega ; \mu_{\Lambda}^{D}(\omega) \in A\right\}\right) .
$$

It is not difficult to show that the set appearing in the right hand side of $(5)$ is measurable (see for instance [8]), so that $\widetilde{P}_{A}$ is well defined. Let now $C\left(\left[a_{0}, b\right]\right)$ be the space of real continuous functions on $\left[a_{0}, b\right]$ and $G_{+}\left(G_{-}\right) \subset C\left(\left[a_{0}, b\right]\right)$ be the set of nondecreasing, nonpositive (nonincreasing, nonnegative) real continuous functions on $\left[a_{0}, b\right]$. To study the behaviour in $\Lambda$ of the measure $\widetilde{P}_{\Lambda}$ we need the following two results:

Lemma 1. Assume that the function $\varphi$ in Assumption (B) satisfies: $\varphi(x) \leqq$ $\exp \left(-x^{(d+\varepsilon)}\right), \varepsilon>0$, for all sufficiently large $x$. (Here $d$ denotes the dimension of $\mathbb{R}^{d}$.) Let $\left\{\Lambda_{n}\right\}_{n \in \mathbb{N}}$ be a sequence of cubes of size $n$ centered at $x=0$. Then for any $g \in G_{-}$(respectively $\left.G_{+}\right)$:

$$
F(g)=\lim _{n \rightarrow+\infty}\left|\Lambda_{n}\right|^{-1} \ln E\left\{\exp \left(\left\langle g, \mu_{\Lambda_{n}}^{D}(\omega)\right\rangle\left|\Lambda_{n}\right|\right)\right\}
$$

exists and it is a convex function on $G_{-}\left(\right.$respectively $\left.G_{+}\right)$. The symbol $<,>$denotes the duality between $C\left(\left[a_{0}, b\right]\right)$ and $M_{a_{0}, b}^{+, k}$. 
Proof. Once the existence of the limit is proved convexity follows from the Hölder inequality. Let us prove existence for $g \in G_{+}$. The case $g \in G_{-}$is similar. Let for any $\Lambda \supset C_{0}$.

$$
F_{A}(g)=E\left\{\exp \left(\left\langle g, \mu_{A}^{D}(\omega)\right\rangle|\Lambda|\right)\right\} .
$$

Using the monotonicity in $\Lambda$ of the eigenvalues $\lambda_{k}\left(H_{\Lambda}^{D}(\omega)\right)$ of $H_{\Lambda}^{D}(\omega)$ (see e.g. [19]):

$$
\lambda_{k}\left(H_{\Lambda}^{D}(\omega)\right) \leqq \lambda_{k}\left(H_{\Lambda^{\prime}}^{D}(\omega)\right) \text { if } \Lambda^{\prime} \subset \Lambda,
$$

we have:

$$
F_{\Lambda}(g) \leqq F_{\Lambda^{\prime}}(g), \text { if } \Lambda^{\prime} \subset \Lambda .
$$

Furthermore using (B) if $\Lambda_{1}$ and $\Lambda_{2}$ are two disjoint cubes at distance $d\left(\Lambda_{1}, \Lambda_{2}\right)=R_{0}$, one has:

$$
F_{A_{1} \cup A_{2}}(g) \leqq F_{A_{1}}(g) \cdot\left(F_{A_{2}}(g)+\exp \left(|g|_{\infty} k\left|\Lambda_{2}\right|\right) \varphi\left(R_{0}\right)\right) .
$$

Using now the assumption $\varphi\left(R_{0}\right) \leqq \exp \left(-R_{0}^{(d+\varepsilon)}\right)$ for $R_{0}$ sufficiently large and the same type of arguments used in satistical mechanics to prove the existence of the entropy for tempered potentials (see e.g. [22]), we get the statement.

Lemma 2. Let $V_{\omega}$ satisfy (A) and (B) and let for $f \in G_{+} \cup G_{-}, Y_{n}(\omega)=\left\langle f, \mu_{\Lambda_{n}}^{D}(\omega)\right\rangle$. Then $Y_{n}(\omega)$ converge geometrically as $n \rightarrow+\infty$ to $\left\langle f, \mu_{\infty}\right\rangle$, i.e. for all $\delta>0$ and all sufficiently large $n$ :

$$
P\left(\left|Y_{n}(\omega)-\left\langle f, \mu_{\infty}\right\rangle\right|>\delta\right) \leqq \exp \left(-\left|\Lambda_{n}\right| M(\delta)\right),
$$

where $M(\delta)>0$.

Proof. Assume for definiteness $f \in G_{+}$. Fix $\delta>0$, and let $n$ and $n_{0}$ be such that:

i) $E\left\{Y_{n_{0}}\right\} \leqq\left\langle f, \mu_{\infty}\right\rangle+\delta / 2$,

ii) $n / n_{0}$ is even.

We then divide the cube $\Lambda_{n}$ in $\left(n / n_{0}\right)^{d}$ subcubes $\Lambda_{n 0_{0}}^{(i)}$. Using (7), the assumption $f \in G_{+}$ and the Chebyshev inequality for the exponential function, we get for any $\eta>0$ :

$$
P\left(Y_{n} \geqq\left\langle f, \mu_{\infty}\right\rangle+\delta\right) \leqq \exp \left\{-\left|\Lambda_{n}\right| \eta\left(\left\langle f, \mu_{\infty}\right\rangle+\delta\right)\right\} \cdot E\left\{\prod_{i} \exp \left(Y_{n_{0}}^{(i)}\left|\Lambda_{n_{0}}\right| \eta\right)\right\},
$$

where $Y_{n_{0}}^{(i)}(\omega)=\left\langle f, \mu_{\Lambda_{n_{0}}^{(0)}}^{D}(\omega)\right\rangle$. To estimate the expectation on the right hand side of (10), we first rearrange the product as:

$$
\prod_{i} \exp \left(Y_{n_{0}}^{i}\left|\Lambda_{n_{0}}\right| \eta\right)=\prod_{\substack{i \\ i_{1} \text { even }}} \exp \left(Y_{n_{0}}^{i}\left|\Lambda_{n_{0}}\right| \eta\right) \prod_{\substack{i \\ i_{1} \text { odd }}} \exp \left(Y_{n_{0}}^{i}\left|\Lambda_{n_{0}}\right| \eta\right)
$$

where $i_{1}=1 \ldots n / n_{0}$ labels from the left to the right the rows of cubes $\Lambda_{n_{0}}^{(i)}$ perpendicular to the first axis, and then apply the Schwartz inequality. By repeating 
this in all directions we obtain:

$$
E\left\{\prod_{i} \exp \left(Y_{n_{0}}^{i}\left|\Lambda_{n_{0}}\right| \eta\right)\right\} \leqq E\left\{\prod_{\substack{i \\ i_{j} \text { odd }, j=1 \ldots d}} \exp \left(Y_{n 0}^{i}\left|\Lambda_{n_{0}}\right| \eta 2^{d}\right)\right\} .
$$

Using (B) we can bound the right hand side of (11) by:

$$
\left\{E\left\{\exp \left(Y_{n_{0}}\left|\Lambda_{n_{0}}\right| \eta 2^{d}\right)\right\}+\varphi\left(n_{0}\right) \exp \left(|f|_{\infty} \eta\left|\Lambda_{n_{0}}\right| k 2^{d}\right)\right\}^{\left(n / 2 n_{0}\right)^{d}}
$$

Let now $v_{n_{0}}$ be the measure on $\mathbb{R}$ given by $v_{n_{0}}(A)=P\left(Y_{n_{0}}(\omega) \in A\right), A$ a measurable set in $\mathbb{R}$, and let

$$
\tilde{v}_{n_{0}}=\left\{v_{n_{0}}+\varphi\left(n_{0}\right) \delta_{\{k|f| \infty\}}\right\} \cdot\left\{1+\varphi\left(n_{0}\right)\right\}^{-1} .
$$

Here $\delta_{\{x\}}$ denotes the Dirac measure centered at $x$. Then we can rewrite (12) as:

$$
\left[\left\{\int d \tilde{v}_{n_{0}}(x) \exp \left(\left|\Lambda_{n_{0}}\right| \eta 2^{d} x\right)\right\} \cdot\left\{1+\varphi\left(n_{0}\right)\right\}\right]^{\left(n / 2 n_{0}\right)^{d}} .
$$

Inserting (13) in (10) and maximizing with respect to $\eta \geqq 0$ we get:

$$
\begin{gathered}
P\left(Y_{n} \geqq\left\langle f, \mu_{\infty}\right\rangle+\delta\right) \leqq \exp \left\{\left[-\left|\Lambda_{n}\right|\left|\Lambda_{n_{0}}\right|^{-1} 2^{-d}\right] .\right. \\
\left.\cdot\left[\sup _{\eta \geqq 0}\left\{\eta\left(\left\langle f, \mu_{\infty}\right\rangle+\delta\right)-\ln \int d \tilde{v}_{n_{0}}(x) \exp (\eta x)\right\}-\ln \left(1+\varphi\left(n_{0}\right)\right)\right]\right\} .
\end{gathered}
$$

Let us now choose $n$ so large that we can choose $n_{0}$ such that:

$$
\int d \tilde{v}_{n_{0}}(x) x \leqq\left\langle f, \mu_{\infty}\right\rangle+\frac{2}{3} \delta
$$

with this choice

$$
\sup _{\eta \geqq 0}\left\{\eta\left(\left\langle f, \mu_{\infty}\right\rangle+\delta\right)-\ln \left(\int d \tilde{v}_{n_{0}}(x) e^{\eta x}\right)\right\} \equiv I_{n_{0}}\left(\left\langle f, \mu_{\infty}\right\rangle+\delta\right)
$$

is positive since it is the Cramer transform of the measure $\tilde{v}_{n_{0}}$ computed in a point strictly bigger than $\int d \tilde{\nu}_{n_{0}}(x) x$ (see e.g. [2]). Furthermore, since $\tilde{v}_{n_{0}} \rightarrow \delta_{\{\langle f, \mu \infty\rangle\}}$ weakly, it is easy to check that $I_{n_{0}}\left(\left\langle f, \mu_{\infty}\right\rangle+\delta\right)$ is bounded away from zero uniformly in $n_{0}$. This is turn implies that for large $n_{0}$ which depends only on $\delta, \varepsilon$, $f$, the square bracket in (14) is positive, i.e.

$$
P\left(Y_{n} \geqq\left\langle f, \mu_{\infty}\right\rangle+\delta\right) \leqq e^{-\left|\Lambda_{n}\right| M(\delta)},
$$

$M(\delta)>0$ for all sufficiently large $n$.

The case $f \in G_{-}$goes analogously if instead of (7) one uses the inequality: $\lambda_{k}\left(H_{\Lambda}^{D}(\omega)\right) \geqq \lambda_{k}\left(H_{\Lambda}^{N}(\omega)\right) \geqq \lambda_{k}\left(H_{\Lambda_{1} \cup \Lambda_{2}}^{N}(\omega)\right)$ for all $\Lambda_{1}, \Lambda_{2}$ such that $\Lambda_{1} \cup \Lambda_{2} \subset \Lambda$ and $\Lambda \backslash \Lambda_{1} \cup \Lambda_{2}$ has zero Lebesgue measure (see e.g. [19]). Similar arguments also give the same bound on $P\left(Y_{n} \leqq\left\langle f, \mu_{\infty}\right\rangle-\delta\right)$ thus concluding the proof of the lemma.

Using the two Lemmas it is now easy to establish the main result. We first extend the function $F: G_{+} \cup G_{-} \rightarrow \mathbb{R}$ given by Lemma 1 to all $C\left(\left[a_{0}, b\right]\right)$ by setting:

$$
F(f)=\lim _{n \rightarrow+\infty}\left|\Lambda_{n}\right|^{-1} \ln E\left\{\exp \left(\left\langle f, \mu_{\Lambda_{n}}^{D}(\omega)\right\rangle\left|\Lambda_{n}\right|\right)\right\} \forall f \in C\left(\left[a_{0}, b\right]\right) \backslash G_{+} \cup G_{-} .
$$

Clearly the above limit is well defined and the new function one obtains is convex 
from $C\left(\left[a_{0}, b\right]\right)$ to $\mathbb{R}$. We then set for each $\mu \in M_{a_{0}, b}^{+, k}$

$$
\lambda(\mu)=\sup _{f \in C\left(\left[a_{0}, b\right]\right)}\{\langle f, \mu\rangle-F(f)\} .
$$

The next result tells us that $\lambda(\mu)$ has a unique absolute minimum at $\mu_{\infty}$, where it is zero.

Theorem 1. In the hypothesis of Lemma 1 the following holds:

i) $\inf _{\mu \in M_{a, b, k}^{+, k}} \lambda(\mu)=\lambda\left(\mu_{\infty}\right)=0$,

ii) if $\mu \in M_{a_{0}, b}^{+, k}$ and $\mu \neq \mu_{\infty}$, then $\lambda(\mu)>0$.

Proof. By the Jensen inequality $F(f) \geqq\left\langle f, \mu_{\infty}\right\rangle \forall f \in C\left(\left[a_{0}, b\right]\right)$; hence $\lambda\left(\mu_{\infty}\right) \leqq 0$. Thus it is sufficient to prove that for any $\mu \lambda(\mu) \geqq 0$ and that if $\mu \neq \mu_{\infty}, \lambda(\mu)>0$. Clearly

$$
\lambda(\mu) \geqq \sup _{t \in \mathbb{R}}\{t\langle f, \mu\rangle-F(t f)\} \quad \forall f \in G_{+} \cup G_{-} .
$$

Furthermore from the geometric convergence of $\left\langle f, \mu_{\Lambda_{n}}^{D}(\omega)\right\rangle$ to $\left\langle f, \mu_{\infty}\right\rangle$ and a result of Ellis (see Th. II 5.1 of [5]) it follows that $\sup _{t \in \mathbb{R}}\{t\langle f, \mu\rangle-F(t f)\} \geqq 0, f \in G_{+} \cup G_{-}$, equality holds iff $\langle f, \mu\rangle=\left\langle f, \mu_{\infty}\right\rangle$. The theorem is now proved if we observe that if $\mu \neq \mu_{\infty}$ there exists an $f_{0} \in G_{+} \cup G_{-}$such that $\left\langle f_{0}, \mu\right\rangle \neq\left\langle f_{0}, \mu_{\infty}\right\rangle$ (iff not $\mu$ would coincide with $\mu_{\infty}$ on the polynomials on $\left[a_{0}, b\right]$ and thus by the Weierstrass theorem on all $\left.C\left(\left[a_{0}, b\right]\right)\right)$.

We can now establish an upper bound on the probability for large fluctuations of $\mu_{\Lambda}^{D}(\omega)$ around $\mu_{\infty}$.

Theorem 2. Let $A \subset M_{a_{0}, b}^{+, k}$ be closed and set $\Lambda(A)=\inf \lambda(\mu)$. Then in the hypothesis of Lemma 1, we have:

$$
\lim _{n \rightarrow+\infty}\left|\Lambda_{n}\right|^{-1} \ln \widetilde{P}_{\Lambda_{n}}(A) \leqq-\Lambda(A),
$$

and $\Lambda(A)>0$ iff $\mu_{\infty} \notin A$.

Proof. Since $M_{a_{0}, b}^{+, k}$ is compact in the weak-*-topology, $A$ is compact. Furthermore it is easily seen that $\lambda(\mu)$ is lower semicontinuous so that inf $\lambda(\mu)=\lambda\left(\mu_{0}\right)$ for some $\mu_{0} \in A$. Thus the second part of the theorem follows from Theorem 1. Using now Chebyshev's inequality we obtain for all $f \in C\left(\left[a_{0}, b\right]\right)$ :

$$
\begin{aligned}
& \tilde{P}_{\Lambda_{n}}(A) \leqq P\left(\left\langle f, \mu_{\Lambda_{n}}^{D}(\omega)\right\rangle \geqq \inf \langle f, \mu\rangle\right) \\
& \quad \leqq \exp \left\{-\left|\Lambda_{n}\right|\left[\inf _{\mu \in A}\langle f, \mu\rangle-\left|\Lambda_{n}\right|^{-1} \ln E\left\{\exp \left(\left\langle f, \mu_{\Lambda_{n}}^{D}(\omega)\right\rangle\left|\Lambda_{n}\right|\right)\right\}\right]\right\} .
\end{aligned}
$$

Taking the logarithm, dividing by $\left|\Lambda_{n}\right|$ and passing to the limit $n \rightarrow+\infty$ we get:

$$
\lim _{n \rightarrow+\infty}\left|\Lambda_{n}\right|^{-1} \ln \widetilde{P}_{\Lambda_{n}}(A) \leqq-\inf _{\mu \in A}\{\langle f, \mu\rangle-F(f)\} \forall f \in C\left(\left[a_{0}, b\right]\right) .
$$


The result now follows by taking the supremum over $f$ of the right hand side of (18) and observing that since $\langle f, \mu\rangle-F(f)$ is convex in $\mu$ and concave in $f$ and furthermore $A$ is compact:

$$
\sup _{f \in C\left(\left[a_{0}, b\right]\right)} \inf _{\mu \in A}\{\langle f, \mu\rangle-F(f)\}=\inf _{\mu \in A} \sup _{f \in C\left(\left[a_{0}, b\right]\right)}\{\langle f, \mu\rangle-F(f)\},
$$

by a result of Sion [23].

A simple application of the above theorem is to compute the probability of events of the form $\left\{\omega \in \Omega ; \rho\left(E, H_{\Lambda}^{D}(\omega)\right) \geqq x|\Lambda|\right\}$. For this we let for any fixed $E>a_{0}$ :

$$
F(t)=\lim _{n \rightarrow+\infty}\left|\Lambda_{n}\right|^{-1} \ln E\left\{\operatorname { e x p } \left(t \rho\left(E, H_{\Lambda_{n}}^{D}(\omega)\right\} \quad t \in \mathbb{R} .\right.\right.
$$

According to Lemma 1 the above limit exists for all $t \in \mathbb{R}$ and it is a convex function of $t$. Let $\lambda(x)=\sup _{t \in \mathbb{R}}\{t x-F(t)\}$ be its Legendre transform and dom $\lambda=\{x ; \lambda(x)<+\infty\}$. Since $F(t)$ is defined for all $t \in \mathbb{R}, \operatorname{dom} \lambda$ is a closed convex set and $F(t)=\sup _{x \in \mathbb{R}}\{t x$ $-\lambda(x)\}$ (see e.g. [20]). It is also not difficult to see that dom $\lambda$ has nonempty interior $(\operatorname{dom} \lambda)^{\text {int }}$. Let now $A(x, E)=\left\{\mu \in M_{a_{0}, E}^{+, k} ; \mu\left(\left[a_{0}, E\right]\right) \geqq x\right\}$. Clearly $A(x, E)$ is compact in $M_{a_{0}, E}^{+, k}$. Hence from the above theorem we get for all $x \in(\operatorname{dom} \lambda)^{\text {int }}$ with $x \geqq \rho_{\infty}(E)$ :

$\lim P\left(\omega ; \rho\left(E, H_{\Lambda}^{D}(\omega)\right) \geqq x|\Lambda|\right) \leqq-\quad \inf \lambda(\mu) \leqq \inf \sup (t y-F(t))=\lambda(x)$, since $\lambda$

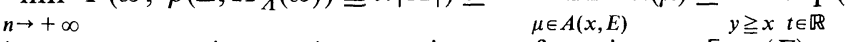
is a monotone increasing continuous function on $\left[\rho_{\infty}(E), \infty\right) \cap \operatorname{dom} \lambda$ (see [20]). It is an interesting question to decide whether the upper bound provided by Theorem 2 is optimal in the sense that $\lim 1 /\left|\Lambda_{n}\right| \ln \widetilde{P}_{A_{n}}(A) \geqq-\Lambda(A)$. The following theorem says that this is the case, at least for simple events of the type $\left\{\omega ; \rho\left(E, H_{\Lambda}^{D}(\omega)\right) \geqq\right.$ $|\Lambda| x\}$, if one assumes a stronger independence property of the random field $V_{\omega}$. In the following we use without comment the notations $F(t), \lambda(x)$ for the functions we have just defined.

Theorem 3. Assume that the $\sigma$-algebras $\sum_{C_{i}}, \sum_{c_{j}} i, j \in Z^{d}$ are independent for all $i \neq j$. Fix $E>a_{0}$. Then for all $x \in(\operatorname{dom} \lambda)^{\text {int }}$ with $x \geqq \rho_{\infty}(E)$ :

$$
\lim _{n \rightarrow+\infty} \frac{1}{\left|\Lambda_{n}\right|} \ln P\left(\rho\left(E, H_{\Lambda_{n}}^{D}(\omega)\right) \geqq x\right)=-\lambda(x) .
$$

Proof. We only need to prove a lower bound. Let us fix $n_{0} \in \mathbb{N}$ and let $N$ be the maximum number of disjoint hypercubes $\Lambda_{n_{0}}^{(i)}$ of size $n_{0}$ strictly contained in $\Lambda_{n}$. Clearly $\left|\Lambda_{n}\right|^{-1} N \rightarrow\left|\Lambda_{n_{0}}\right|$ as $n \rightarrow+\infty$. Then using (7):

$$
\frac{1}{\left|\Lambda_{n}\right|} \ln P\left(\rho\left(E, H_{\Lambda_{n}}^{D}(\omega)\right) \geqq x\left|\Lambda_{n}\right|\right) \geqq \frac{1}{\left|\Lambda_{n}\right|} \ln P\left(\sum_{i}^{N} \rho\left(E, H_{\Lambda_{n_{0}}^{(i)}}^{D}(\omega)\right)\left|\Lambda_{n_{0}}\right|^{-1} \geqq \frac{\left|\Lambda_{n}\right|}{\left|\Lambda_{n}\right|} x\right)
$$

The random variables $\rho\left(E, H_{\Lambda_{n_{0}}^{(i)}}^{D}(\omega)\right)\left|\Lambda_{n_{0}}\right|^{-1}$ are now independent by assumption so 
that we can apply standard large deviation results (see e.g. Azencott [2]) to get:

$$
\lim _{n \rightarrow+\infty} \frac{1}{\left|\Lambda_{n}\right|} \ln P\left(\rho\left(E, H_{\Lambda_{n}}^{D}(\omega)\right) \geqq\left|\Lambda_{n}\right| x\right) \geqq-\sup _{t \in \mathbb{R}}\left(t x-\frac{1}{\left|\Lambda_{n o}\right|} \ln E\left\{\exp t \rho\left(E, H_{\Lambda_{n}}^{D}(\omega)\right)\right\}\right) .
$$

Since $n_{0}$ was arbitrary it remains to prove that

$$
\lambda_{n_{0}}(x) \equiv \sup _{t \in \mathbb{R}}\left\{t x-\frac{1}{\left|\Lambda_{n_{0}}\right|} \ln E\left\{\exp t \rho\left(E, H_{\Lambda_{n_{0}}}^{D}(\omega)\right\}\right\} \equiv \sup _{t \in \mathbb{R}}\left(t x-F_{n_{0}}(t)\right)\right.
$$

converges to $\lambda(x)$ for all $x \in(\operatorname{dom} \lambda)^{\text {int }}$ as $n_{0} \rightarrow+\infty$. Using once again (3) one has by subadditivity:

$$
\begin{aligned}
& \text { i) } \lim _{n_{0} \rightarrow+\infty} \lambda_{n_{0}}(x)=\inf _{n_{0}} \lambda_{n_{0}}(x) \equiv \bar{\lambda}(x), \\
& \text { ii) } F(t)=\sup _{n_{0}} F_{n_{0}}(t)=\lim _{n_{0} \rightarrow+\infty} F_{n_{0}}(t) .
\end{aligned}
$$

Furthermore, taking the Legendre transform:

$$
F_{n_{0}}(t)=\sup _{x}\left\{t x-\lambda_{n_{0}}(x)\right\} \leqq \sup _{x}\{t x-\bar{\lambda}(x)\} . \quad \forall n_{0} \in \mathbb{N},
$$

which implies: $F(t) \leqq \sup _{x}\{t x-\bar{\lambda}(x)\}$.

On the other hand:

$F_{n_{0}}(t)+\lambda_{n_{0}}(x) \geqq t x \forall t, x \in \mathbb{R}$, so that, passing to the limit $n_{0} \rightarrow+\infty: F(t)+\bar{\lambda}(x) \geqq$ $t x \forall t, x \in \mathbb{R}$. Hence $F(t)=\sup _{x}\{t x-\bar{\lambda}(x)\}$. Thus $\bar{\lambda}(x)$ as the pointwise limit of convex functions is convex with Legendre transform identical to that of $\lambda(x)$. It follows (see Rockafeller [20]) that $\bar{\lambda}(x)=\lambda(x)$ for all $x \in(\operatorname{dom} \lambda)^{\mathrm{int}}$.

\section{Section 3. Lifshitz Singularity}

In this section we examine the behaviour of the $\operatorname{IDS} \rho_{\infty}(\lambda)$ as $\lambda \rightarrow 0^{+}$for non-negative random potentials $V_{\omega}$ (i.e., $V_{\omega}(x) \geqq 0 \forall x \in \mathbb{R}^{d}$ a.e). Our main result is the following:

Theorem 4. Let $V_{\omega}$ be an almost surely non-negative random field on $\mathbb{R}^{d}$ which satisfies (A) and (B). Assume that $E\left(\left|\left\{x \in C_{0 i} V_{\omega}(x)=0\right\}\right|\right)=p<1$. Then:

$$
\lim _{\lambda \rightarrow 0^{+}}-\lambda^{d / 2} \ln \rho_{\infty}(\lambda) \geqq k>0
$$

for some positive constant $k$.

Proof. From inequality (4) and the positivity of $V_{\omega}$, we get for any $\Lambda \supset C_{0}$,

$$
\rho_{\infty}(\lambda) \leqq|\Lambda|^{-1} \rho\left(\lambda,-\Delta_{\Lambda}^{N}\right) P\left(\lambda_{1}\left(H_{\Lambda}^{N}(\omega)\right)<\lambda\right) .
$$

We now choose $\Lambda=\Lambda(\lambda)$ to be the cube in $\mathbb{R}^{d}$ centred at $x=0$ of size $L \equiv L(\alpha, \lambda)=$ $\pi(1+\alpha)^{-1 / 2} \lambda^{-1 / 2}$, where $\alpha$ is a positive constant, which will be fixed later on. With 
this choice we compute:

$$
\lambda_{2}\left(-\Delta_{\Lambda(\lambda)}^{N}\right)=\lambda(1+\alpha) .
$$

Furthermore using a lower bound on the lowest eigenvalue of positive selfadjoint operators due to Thirring [24] (see also [19]) we obtain $\forall \Lambda \subset \mathbb{R}^{d}$

$$
\left.\lambda_{1}\left(-\Delta_{\Lambda}^{N}+V_{\omega}+\alpha \lambda\right) \geqq \min \left(\lambda_{2}\left(-\Delta_{\Lambda}^{N}\right),\left\{\int_{\Lambda} d x\left|\psi_{0}(x)\right|^{2}\left(V_{\omega}(x)+\alpha \lambda\right)^{-1}\right)\right\}^{-1}\right),
$$

where $\psi_{0} \in L^{2}(\Lambda)$ is the normalized ground state wave function of $-\Delta_{\Lambda}^{N}$, i.e. $\psi_{0}(x)=$ $|\Lambda|^{-1 / 2} \forall x \in \Lambda$. Equations (22) and (23) together imply:

$$
P\left(\lambda_{1}\left(H_{\Lambda}^{N}(\omega)\right)<\lambda\right) \leqq P\left(|\Lambda(\lambda)|^{-1} \lambda \int_{\Lambda(\lambda)} d x\left(V_{\omega}(x)+\alpha \lambda\right)^{-1} \geqq(1+\alpha)^{-1}\right) .
$$

Let now for $\lambda_{0}>0, \xi\left(\lambda, \lambda_{0}, \omega\right)=|\Lambda(\lambda)|^{-1} \int_{\Lambda(\lambda)} d x\left(V_{\omega}(x)+\alpha \lambda_{0}\right)^{-1} \lambda_{0}$.

The same argument used in the proof of Lemma 2 shows that the random variable $\xi\left(\lambda, \lambda_{0}, \omega\right)$ converges geometrically to $\lim _{\lambda \rightarrow 0^{+}} E\left\{\xi\left(\lambda, \lambda_{0}, \omega\right)\right\}=E \int_{C_{0}} d x \lambda_{0}\left(V_{\omega}(x)+\right.$ $\left.\lambda_{0} \alpha\right)^{-1}$ as $\lambda \rightarrow 0^{+}$. Furthermore by the dominated convergence theorem $E\left\{\int_{C_{0}} d x\left(V_{\omega}(x)+\right.\right.$ $\left.\left.\lambda_{0} \alpha\right)^{-1} \lambda_{0}\right\}$ converges to $\alpha^{-1} p$ as $\lambda_{0} \rightarrow 0^{+}$. These two results together imply that if $(\alpha+1)^{-1}>\alpha^{-1} p$, i.e. $\alpha>p(1-p)^{-1}$, and if $\lambda_{0}$ is such that $E\left\{\int_{C_{0}} d x \lambda_{0}\left(V_{\omega}(x)+\alpha \lambda_{0}\right)^{-1}\right\}<$ $(1+\alpha)^{-1}$, we can find a constant $M(\alpha)$ greater than zero such that for all sufficiently small $\lambda$ :

$$
\begin{aligned}
& P\left(|\Lambda(\lambda)|^{-1} \int_{\Lambda(\lambda)} d x \lambda\left(V_{\omega}(x)+\alpha \lambda\right)^{-1} \geqq(1+\alpha)^{-1}\right) \\
& \quad \leqq P\left(\xi\left(\lambda, \lambda_{0}, \omega\right) \geqq(1+\alpha)^{-1}\right) \leqq \exp (-M(\alpha)|\Lambda(\lambda)|) \\
& \quad=\exp \left(-M(\alpha) \lambda^{-d / 2} \pi^{d}(1+\alpha)^{-2 / d}\right) .
\end{aligned}
$$

The result now follows from (21) observing that by Weyl's result (see e.g. [19]) $|\Lambda(\lambda)|^{-1} \rho\left(\lambda,-\Delta_{\Lambda(\lambda)}^{N}\right) \leqq$ const $\lambda^{d / 2}$.

As in the case of the large deviations for the $\operatorname{IDS} \rho_{\infty}(\lambda)$, we can strengthen the above result if we assume that the $\sigma$-algebras $\Sigma_{\Lambda_{i}}$ generated by disjoint regions $\Lambda_{i}$ are independent. For this let $\gamma(d)$ be the lowest eigenvalue of the Dirichlet Laplacian $-\Delta^{D}$ on the unit ball $B_{1}$ in $\mathbb{R}^{d}$ and let $\tau_{d}=\left|B_{1}\right|$. Then we have:

Theorem 5. In addition to the hypothesis of Theorem 4 assume that the $\sigma$-algebras $\Sigma_{C_{i}}, \Sigma_{C_{j}} i, j \in Z^{d}$ are independent if $i \neq j$. Suppose furthermore that $P\left(\int_{C_{0}} V_{\omega}(x) d x=0\right)=p$ satisfies: $0<p<1$. Then:

$$
\lim _{\lambda \rightarrow 0^{+}}-\lambda^{d / 2} \ln \left\{\rho_{\infty}(\lambda)\right\} \leqq \ln \left\{p^{-1}\right\}(\gamma(d))^{d / 2} \tau_{d} .
$$

Proof. From (4) we have:

$$
\rho_{\infty}(\lambda) \geqq|\Lambda|^{-1} E\left\{\rho\left(\lambda, H_{\Lambda}^{D}(\omega)\right)\right\} \geqq|\Lambda|^{-1} P\left(\lambda_{1}\left(H_{\Lambda}^{D}(\omega)\right) \leqq \lambda\right)
$$


for any $\Lambda \supset C_{0}$. Let now $B_{\lambda}$ be the ball in $\mathbb{R}^{d}$ of radius $R(\lambda)=\left\{\gamma(d) \cdot \lambda^{-1}\right\}^{1 / 2}$ and let us choose in (25) $\Lambda=\Lambda(\lambda)$ as the smallest cube which contains $B_{\lambda}$. We denote by $\left\{C_{i}\right\}_{i=1}^{N(\lambda)}$ the smallest collection of cubes $C_{i}$ which entirely covers $B_{\lambda}$. Then by (7) and the min-max:

$$
\lambda_{1}\left(H_{\Lambda(\lambda)}^{D}(\omega)\right) \leqq \lambda_{1}\left(H_{B_{\lambda}}^{D}(\omega)\right) \leqq \lambda_{1}\left(-\Delta_{B_{\lambda}}^{D}\right)+\int_{B_{\lambda}} d x\left|\psi_{0}(x)\right|^{2} V_{\omega}(x),
$$

where $\psi_{0} \in L^{2}\left(B_{\lambda}\right)$ is the ground state wave function of $\Delta_{B_{\lambda}}^{D}$. A direct computation gives $\lambda_{1}\left(-\Delta_{B_{\lambda}}^{D}\right)=\lambda$ which together with (26) and (25) implies:

$$
\begin{aligned}
& \rho_{\infty}(\lambda) \geqq|\Lambda(\lambda)|^{-1} P\left(\int_{B_{\lambda}} d x V_{\omega}(x)=0\right) \geqq\left\{2 \gamma(d) \lambda^{-1}\right\}^{-d / 2} P\left(\sum_{1}^{N(\lambda)} \int_{C_{i}} d x V_{\omega}(x)=0\right) \\
& =\left\{2 \gamma(d) \lambda^{-1}\right\}^{-d / 2} \exp \left(-N(\lambda) \ln \left\{p^{-1}\right\}\right)
\end{aligned}
$$

The theorem is now proved, noting that

$N(\lambda)\left|B_{\lambda}\right|^{-1}=N(\lambda) \gamma(d)^{-d / 2} \lambda^{d / 2} \tau_{d}^{-1}$ converges to one as $\lambda \rightarrow 0^{+}$.

\section{Examples}

We conclude this note with a discussion of the results of Theorems 4 and 5 in two examples which arise in models of quantum disordered systems.

Example 1. Let $\xi_{\omega}(x), x \in \mathbb{R}^{d}$, be a metrically transitive Gaussian random field with zero mean and unit variance such that

$$
E\left\{\xi_{\omega}(x) \xi_{\omega}(0)\right\}=\eta(x) \text { is integrable, } \eta \in L^{1}\left(\mathbb{R}^{d}\right),
$$

and Riemannian approximable, i.e.

$$
\lim _{a \rightarrow 0^{+}} \sum_{i \in Z^{d}} a^{d} \eta(a i)=\int_{\mathbb{R}^{d}} d x \eta(x) .
$$

Let also $F: \mathbb{R}^{d} \rightarrow \mathbb{R}$ be a locally bounded positive real function on $\mathbb{R}^{d}$, polynomially bounded at infinity and set $V_{\omega}(x)=F\left(\xi_{\omega}(x)\right)$.

It is not difficult to see that the random field $V_{\omega}$ satisfies (A) but not in general (B) (see e.g. [7] for a discussion of the $\varphi$-mixing condition for Gaussian processes). In the next theorem we prove that nevertheless a result similar to Theorem 4 holds.

Theorem 6. Let $\rho_{\infty}(\lambda)$ be the IDS arising from the random field $V_{\omega}$. Then:

i) if $\{\{x ; F(x)=0\} \mid=0$

$$
\lim _{\lambda \rightarrow 0^{+}}-\lambda^{d / 2} \ln \left\{\left(P\left(V_{\omega}(0)<\lambda^{s}\right)\right)^{-1}\right\} \ln \rho_{\infty}(\lambda) \geqq 2 \pi^{d} q^{-1} d^{d / 2}(d+2)^{-(d+2) / 2}
$$

for all $s<1$, where $q=\int_{\mathbb{R}^{d}} d x \eta(x)$.

ii) If $|\{x ; F(x)=0\}|>0$ :

$$
\lim _{\lambda \rightarrow 0^{+}}-\lambda^{d / 2} \ln \left\{\rho_{\infty}(\lambda)\right\} \geqq \pi^{d / 2} \sup _{\alpha>0}(1+\alpha)^{-d / 2} G(\alpha) q^{-1},
$$

where $G(\alpha)=\sup _{t \geqq 0}\left\{t(1+\alpha)^{-1}-\ln \left\{\exp \left(\alpha^{-1} t\right) p+1-p\right\}\right\}, p=P\left(V_{\omega}(0)=0\right)>0$. 
Proof. From the proof of Theorem 4 we have:

$$
\rho_{\infty}(\lambda) \leqq k \lambda^{d / 2} P\left(|\Lambda(\lambda)|^{-1} \int_{\Lambda(\lambda)} d x \lambda\left(V_{\omega}(x)+\alpha \lambda\right)^{-1} \geqq(1+\alpha)^{-1}\right),
$$

where $\Lambda(\lambda)$ is the cube of size $L(\alpha, \lambda)=\pi(1+\alpha)^{-1 / 2} \lambda^{-1 / 2}$ and $k$ a positive constant. Chebyshev's inequality for the exponential function gives for any $t \geqq 0$;

$$
\begin{aligned}
& P\left(|\Lambda(\lambda)|^{-1} \int_{\Lambda(\lambda)} d x \lambda\left(V_{\omega}(x)+\alpha \lambda\right)^{-1} \geqq(1+\alpha)^{-1}\right) \\
& \leqq \exp \left\{-|\Lambda(\lambda)| t(1+\alpha)^{-1}\right\} E\left\{\exp \left(\int_{\Lambda(\lambda)} d x \lambda t\left(V_{\omega}(x)+\alpha \lambda\right)^{-1}\right)\right\} .
\end{aligned}
$$

We can now use recent decoupling inequalities for stationary Gaussian random fields with an integrable correlation function [13] to get:

$$
E\left\{\exp \left(\int_{\Lambda(\lambda)} d x t \lambda\left(V_{\omega}(x)+\alpha \lambda\right)^{-1}\right)\right\} \leqq E\left\{\exp \left(q t \lambda\left(V_{\omega}(0)+\alpha \lambda\right)^{-1}\right)\right\}^{q^{-1}|\lambda(\lambda)|} .
$$

If we insert (30) in (29) and maximize with respect to $t \geqq 0$ we obtain:

$$
P\left(|\Lambda(\lambda)|^{-1} \int_{\Lambda(\lambda)} d x \lambda\left(V_{\omega}(x)+\alpha \lambda\right)^{-1} \geqq(1+\alpha)^{-1}\right) \leqq \exp \left\{-|\Lambda(\lambda)| q^{-1} \tilde{G}(\alpha, \lambda)\right\},
$$

where $\tilde{G}(\alpha, \lambda)=\sup _{t \geqq 0}\left\{t(1+\alpha)^{-1}-\ln \left\{E\left(\exp \left(t \lambda\left[V_{\omega}(0)+\alpha \lambda\right]^{-1}\right)\right)\right\}\right\}$.

For any $s<1$ the estimate:

$$
\begin{aligned}
E\left\{\exp \left(t \lambda\left(V_{\omega}(0)+\alpha \lambda\right)^{-1}\right)\right\} \leqq & \exp \left(t \alpha^{-1}\right) P\left(V_{\omega}(0)<\lambda^{s}\right) \\
& +\exp \left(t \lambda\left(\lambda^{s}+\alpha \lambda\right)^{-1} P\left(V_{\omega}(0)>\lambda^{s}\right)\right.
\end{aligned}
$$

gives:

$$
\begin{aligned}
\widetilde{G}(\alpha, \lambda) \geqq & \sup \left\{t(1+\alpha)^{-1}-\ln \left\{\exp \left(\alpha^{-1} t\right) P\left(V_{\omega}(0) \leqq \lambda^{s}\right)\right.\right. \\
& +\exp \left(t \lambda\left(\lambda^{s}+\alpha \lambda\right)^{-1}\left(1-P\left(V_{\omega}(0) \leqq \lambda^{s}\right)\right)\right\} \equiv G(\alpha, \lambda) .
\end{aligned}
$$

We consider the two cases:

$\lim _{\lambda \rightarrow 0^{+}} P\left(V_{\omega}(0) \leqq \lambda^{s}\right)=p=0$ and $p>0$, separately corresponding to $\mid\left\{x \in \mathbb{R}^{d} ; F(x)\right.$ $=0\} \mid=0$ and $\left|\left\{x \in \mathbb{R}^{d} ; F(x)=0\right\}\right|>0$. In the first case, $p=0$, it is easy to see that:

$$
\lim _{\lambda \rightarrow 0^{+}} G(\alpha, \lambda)\left\{\ln \left(P\left(V_{\omega}(0)<\lambda^{s}\right)^{-1}\right)\right\}^{-1}=\alpha(1+\alpha)^{-1} \quad \forall \alpha>0 .
$$

Thus in this case the statement follows from (31), (33), (34), the definition of $\Lambda(\lambda)$ and $\sup _{\alpha>0}(1+\alpha)^{-(d / 2)-1} \alpha=2 d^{d / 2}(d+2)^{-d+2 / 2}$.

For $p>0$ we explicitly compute:

$$
\lim _{\lambda \rightarrow 0} G(\alpha, \lambda)=\sup _{t \geqq 0}\left\{t(\alpha+1)^{-1}-\ln \left\{\exp \left(\alpha^{-1} t\right) p+1-p\right\}\right\}=G(a) .
$$

Thus the theorem follows from (31), (33), (35) and the definition of $\Lambda(\lambda)$. It is also easy 
to show that in this case

$$
\sup _{\alpha>0}(1+\alpha)^{-d / 2} G(\alpha)>0
$$

Example 2. Let $\ell^{1}\left(L^{p}\right)$ be the Banach space of all measurable real functions on $\mathbb{R}^{d}$ with:

$$
\|f\|_{\ell^{1}\left(L^{p}\right)}=\left.\left.\sum_{i \in Z^{d}}\left|\int_{C_{i}} d x\right| f(x)\right|^{p}\right|^{1 / p}<+\infty .
$$

Let $\left\{\varphi_{i}(\omega)\right\}_{i \in Z^{d}}$ be $\ell^{1}\left(L^{p}\right)$-valued iid random variables, $p$ is as in $(\mathrm{A})$, such that:

i) $\varphi_{0}(\omega, x) \geqq 0$ a.e. and $1>P\left(\varphi_{0}(\omega)=0\right)>0$.

ii) There exist two positive constants $k_{1}, k_{2}$ and a positive random variable $\eta_{0}(\omega)$ with $E\left\{\left|\eta_{0}(\omega)\right|^{p}\right\}<+\infty$, such that:

$k_{2} \eta_{0}(\omega)|x|^{-\alpha} \geqq \varphi_{0}(\omega, x) \geqq k_{1} \eta_{0}(\omega)|x|^{-\alpha}, \alpha>d$ for all $x \in \mathbb{R}^{d}$ with $|x|$ sufficiently large. We then define:

$$
V_{\omega}(x)=\sum_{i \in Z^{d}} \varphi_{i}(\omega, x-i)
$$

From i) and ii) it follows that $V_{\omega}$ is a well defined random field on $\mathbb{R}^{d}$ which satisfies (A).

A typical example is the case where the random variables $\varphi_{i}(\omega)$ are of the form: $\varphi_{i}(\omega, x)=q_{i}(\omega) f(x)$, where $\left\{q_{i}\right\}_{i \in Z^{a}}$ are iid positive random variables with $E\left\{\left|q_{0}(\omega)\right|^{p}\right\}<+\infty$ and $f$ a positive function in $\ell^{1}\left(L^{p}\right)$ such that $f(x) \sim|x|^{-\alpha}$ as $|x| \rightarrow$ $+\infty$. In [9] and [12] we proved that in this situation the spectrum of $-\Delta$ $+\sum_{i} q_{i}(\omega) f(x-i)$ has a band structure and that in dimension greater than 1 it contains the interval $\left(E_{0}, \infty\right)$ for some $E_{0}<+\infty$. For random fields $V_{\omega}$ as given by (36) Theorems 4 and 5 are modified as follows:

Theorem 7. Let $V_{\omega}$ be given by (36) and let $\rho_{\infty}(\lambda)$ be the associated IDS Then:

i) if $\alpha \geqq d+2 \lim _{\lambda \rightarrow 0^{+}}-[\ln \{\lambda\}]^{-1} \ln \left\{\ln \left\{\left(\rho_{\infty}(\lambda)\right)^{-1}\right\}\right\}=d / 2$,

ii) If $d+2>\alpha>d \lim _{\lambda \rightarrow 0^{+}}-[\ln \lambda]^{-1} \ln \left\{\ln \left\{\left(\rho_{\infty}(\lambda)\right)^{-1}\right\}\right\}=d(\alpha-d)^{-1}$.

The proof of this result can be found in [15]; it follows closely the proof of Theorems 4 and 5 and uses for the long-range case $d+2>\alpha>d$, an estimate on $\rho_{\infty}(\lambda)$ proved in $[10]$ of the form:

$$
\rho_{\infty}(\lambda) \leqq k \lambda^{d / 2} P\left(\int_{C_{0}} d x V_{\omega}(x)<\lambda\right),
$$

where $k$ is a positive constant. 
Acknowledgements. We are in debt to S. Albeverio for many constructive comments. One of us (F.M.) would also like to thank G. Jona-Lasinio and E. Scoppola for very stimulating discussions and Professors Zehnder and Albeverio for their kind hospitality at the Mathematisches Institut der RuhrUniversität, Bochum

\section{References}

1. Avron, J., Simon, B. : Almost periodic Schrödinger operators: II. the density of states. Preprint

2. Azencott, R.: Grandes deviations and applications in Ecole d'Eté des Probabilités de Sain-Flour VIII-1978. In: Lecture Notes in Mathematics Vol. 774 Berlin, Heidelberg, New York: Springer 1978

3. Billingsley, P.: Convergence of probability measures. New York: J. Wiley 1968

4. Donsker, M. Varadhan, S. : Asymptotics for the Wiener sausage. Commun. Pure. Appl. Math. 28, 525-565 (1975)

5. Ellis, R. : Large deviations and other limit theorems for a class of dependent random variables with applications to statistical mechanics. Preprint 1981

6. Friedberg, R., Luttinger, J. M. : Density of electronic energy Levels in disordered systems. Phys. Rev. B12, 4460-4474 (1975)

7. Ibragimov, I. A., Linnik, Yu. V.: Independent and Stationary sequences of Random Variables. Groningen 1971

8. Kirsch, W., Martinelli, F.: On the ergodic properties of the spectrum of general random operators. J. Reine Angew. Math. 334, 141-156 (1982)

9. Kirsch, W., Martinelli, F.: On the spectrum of a random Schrödinger operator. Commun. Math. Phys. 85, 329-350 (1982)

10. Kirsch, W., Martinelli, F.: On the density of states of Schrödinger operators with a random potential. J. Phys. A15, 2139-2156 (1982)

11. Kirsch, W., Martinelli, F.: On the essential selfadjointness of stochastic Schrödinger operators, Bochum Preprint 1981

12. Kirsch, W., Martinelli, F.: Some results on the spectra of random Schrödinger operators and their applications to random point interactions models in one and three dimensions, 173, Proceedings of the Inter. Conference "Stochastic methods", Marseille 1981, Lecture Notes in Physics, Springer

13. Klein, A., Landau, L. J., Shucker, D. S. : Decoupling inequalities for stationary gaussian processes. Preprint

14. Lifshitz, I. M. : Energy spectrum structure and quantum states of disordered condensed systems. Sov. Phys. Usp. 7, No 4 (1965)

15. Martinelli, F.: On the density of states of random Schrödinger operators. Thesis in preparation

16. Nakao, S. : On the spectrum distribution of the Schrödinger operator with a random potential. Jpn. J. Math. 3, 111-139 (1977)

17. Pastur, L. A. : Spectra of random selfadjoint operators. Russ. Math. Surv. 28, 1-67 (1973)

18. Pastur, L. A.: On the distribution of the eigenvalues of the Schrödinger equation with a random potential. Funkt. Anal. Pril. 6, No 2, 93-94 (1972)

19. Reed, M., Simon, B. : Methods of modern mathematical physics, Vol. IV, II, New York: Academic Press 1978

20. Rockafeller, T. : Convex Analysis. Princeton, NJ: Princeton 1970

21. Romerio, M., Wreszinski, W. : On the Lifshitz singularity and the tailing in the density of states for random lattice systems, J. Stat. Phys. 21, No 2, 169-179 (1979)

22. Ruelle, D. : Statistical mechanics. New York: Benjamin 1969

23. Sion, M.: On general minimax theorems Pac. J. Math. 8, (1958)

24. Thirring, W.: Vorlesungen über Mathematische Physik. Univ. Wien Lecture Notes

Communicated by B. Simon

Received October 14, 1982; in revised form December 28, 1982 